\title{
Historical Building Inspection using the Unmanned Aerial Vehicle (Uav)
}

\author{
Hanita Yusof ${ }^{1}$, Mustaffa Anjang Ahmad ${ }^{1}$, Aadam Mohammed Taha Abdullah ${ }^{1^{*}}$ \\ ${ }^{1}$ Universiti Tun Hussein Onn Malaysia, 86400 Parit Raja, Batu Pahat, Johor, MALAYSIA \\ *Corresponding Author
}

DOI: https://doi.org/10.30880/ijscet.2020.11.03.002

Received 15 January 2020; Accepted 19 June 2020; Available online 30 June 2020

\begin{abstract}
Building inspection is very much required for all buildings especially those historical ones to maintain the structure condition and the safety of the people around. Visual inspection is commonly conducted using manual descriptive information carried out by the inspector personally. The problem with this technique of assessment is that the time needed to write all the defect description on site and to access difficult area can be hazardous. The aim of performing historical building inspection on Tan Swee Hoe's historical mansion is to evaluate the overall condition of the building with Unmanned Aerial Vehicle (UAV) image assisted inspection and Condition Survey Protocol 1 (CSP1) method. From this drone assisted inspection, it shows that the time spent on site is less than half an hour and the data collected being evaluated with CSP1 method defined that the building is dilapidated. The overall building condition is in red class and require serious attention to avoid any injuries to the visitors. To prevent a possible failure of a building in the coming years, a suitable condition inspection has been carried out to identify the current existing defect so that it would be fixed before further damage to the building and visitors around it.
\end{abstract}

Keywords: UAV Inspection, Historical Building Inspection, Historical Buildings Conservation, Heritage Building Malaysia.

\section{Introduction}

Abandoning historical or heritage building is not a new phenomenon. There are many dilapidated historical buildings that can be seen around Malaysia. Among the biggest concerns was the abandonment which would lead to the destruction that becomes such a waste to see our heritage of woodcarving being destroyed unappreciated and undocumented (Hanita Yusof, 2017). Historical building is usually very old and have a lot of structure failure or defects which can be seen by naked eye or identified by professionals. On different perspective which is seen by the Unmanned Aerial Vehicle (UAV) could improve the observation and inspection of the building. UAV can access hard-to-reach areas since historical building is not safe to be in and probably cannot withstand any load other than itself and could be dangerous for building inspector (Fisher, 2015). Data analysis from the UAV image can identify the level of failure. In order to conserve and use the building without losing the identity and landmark of the building, it has to use the original state of the building as much as it could. Technologically advanced tools such as high definition cameras and UAV enable professionals to analyze such issues rapidly and accurately while reducing operational costs and minimizing safety risks, and when paired with video recording, photography or multi spectral imaging, drones can safely, economically, and efficiently carry out a broad variety of surveying services (Tahar, 2012). 


\section{Literature Review}

Historical building inspection need the special heavy vehicles that requires time and expensive cost (Mader et al., 2016). This requires a highly up-to-date and effective monitoring system for all important constructions public life. Based on many studies it can be stated that the use of UAVs for visual inspections is an alternative to costly photogrammetric measurements and time consuming field measurements (Benaszek et al., 2010; Mader et al., 2016; Al-Kaff et al., 2017).

UAV strengths include high mobility in data collecting and the ability to fly at different heights. High-resolution digital images are useful for UAVs for inspection and inspection of buildings and structures. UAV data acquisition can be successfully used in the energy sector for inventories of power lines and inspecting the state of the energy infrastructure. Utilizing UAVs for inspections of hard-to-reach or hazardous locations plus increases the safety of work (Perritt \& Sprague, 2016).

\subsection{Unmanned Aerial Vehicle (UAV)}

Drones, also referred to as Unmanned Aerial Vehicles (UAVs) in the literature, can be loosely defined as an aircraft without a human pilot aboard. A UAV's flight might be controlled either self-sufficiently by installed PCs or by remote control of a pilot on the ground or in another vehicle. A UAV is normally propelled and recuperated by means of a programmed framework or an outer administrator on the ground. There are a wide variety of UAV shapes, sizes, configurations, characteristics, etc. UAVs may be used for a growing number of applications, such as police surveillance, firefighting, security work (e.g., surveillance of pipelines), surveillance of farms, commercial purposes etc (Srivastava et al., 2017).

Phantom 3 Standard is one of the UAV model that has been used in visual inspection. This drone equipped with Global Positioning System GPS system and has a core design that lifts the centre of gravity closer to the centroid, improving balance and making it more agile and impressively precise. Fig. 2 shows phantom 3 fits with the criteria needed and makes flying remarkably intuitive and easy. From take-off to landing, it's completely under control, responding to commands while automatically handling the most complex aspects of safe and stable flight. Data collection will be used with the camera 1520p high definition image and video basically everything the camera see plus can zoom and still observe clearly. It makes it very easy to fly at $5 \mathrm{~m} / \mathrm{s}$ to high ground approximately $6000 \mathrm{~m}$ above sea level and collect data at that altitude (DJI Phantom, 2015).

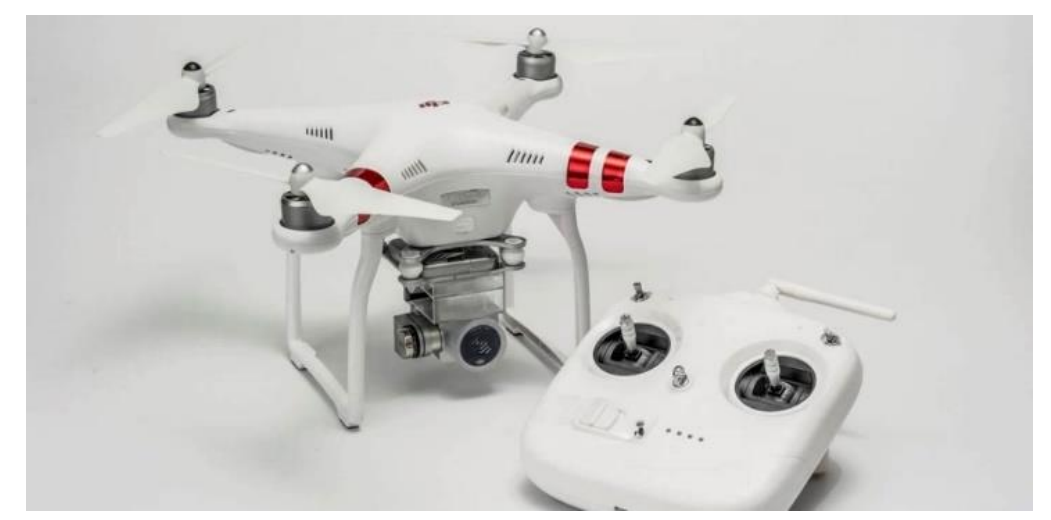

Fig. 2 - Phantom 3 standard (DJI Phantom, 2015)

\subsection{Case study}

Historical building inspection based on UAV can be a good approach in preserving these historical building. Rather than demolishing the whole building out, a simple inspection should be carried out to determine the condition of the building and which part should be taken care first. This study interested in the application of UAV for historical building inspection by preparing the overall condition of the building in order to conserve the building. Most studies in the field of this assessment only focused on common building inspection for maintenance purpose. The objectives of the historical building inspection is to perform a building inspection using UAV and analyse the severity of damage of the building by observing picture and video taken and prepare the overall condition of the building using Condition Survey Protocol. It is significant to demonstrate that the condition rating and safety inspection level of the historical building inspection in this study could be applied widely on any building inspection by applying Condition Survey Protocol (CSP1) matrix. In addition, this technique utilized comparatively nonspecialized inspection method compared to the conventional practice and has advantages on different aspect. The videos and pictures taken identify the damages. With this study, the data taken are used to analyze the overall condition and the severity of damage on the building. 
The scope of study focused on the several stages such as updating procedure, data collection and condition rating. Updating the procedure and inventories involve collecting applicable information through available building inspection spreadsheet and Public Work Department (PWD) standard for observation procedure a building. From figure 1 below all assessment will be done at Tan Swee Hoe's building, Batu Pahat, Johor.

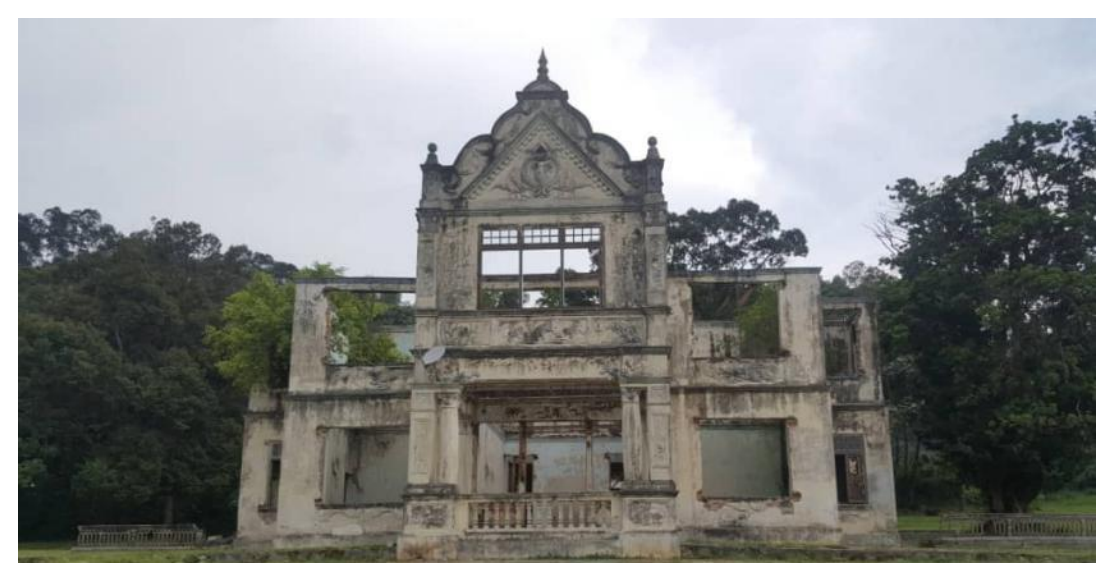

Fig. 1 - Heritage building of Tan Swee Hoe

Survey the state of the building and damage known as dilapidation study. It is a process to identify and record the condition or degree of disability of the building. This study is a practice of documenting the problems and defects of the building based on UAV. The study is used to record and document, a thorough investigation is necessary to determine the condition of the building, the defect and the severity of damage. Documentation in the form of digital and photography need to detect the circumstances and the nature of the materials of the existing building (Kaamin et al., 2016).

\section{Methodology}

Several methods currently exist for building inspection and severity of damage of building depending on building type. Visual inspection is one of the most common procedures for determining condition of building and analysing data..

\subsection{Preparation Stage}

The first steps of this research came with the information of the building itself from district office of Batu Pahat and the authorization to evaluate the building from the officer in charge. Information were also taken from web search such as google, google earth, online newspaper on previous personal evaluation related to main objectives especially due to building defect.

\subsection{Data Collection on Site}

Two different data collections were done on the site visit at the building. The first one is manually collecting data through observation and measurement. The second one is by collecting data by UAV. Firstly the software application is downloaded to be used for drone visual camera to examine camera angle. Fig. 3 shows the sketched flight plan illustrated for the UAV to fly and the angle of camera pointed to the building as required by Agisoft Photoscan software for best data analysis result.

Main preparation of data collected is listed below:

i. Checking the stabilization of drone.

ii. Estimating time flight and battery supplied.

iii. Setting the takeoff and landing area for the drone.

iv. Dividing section into 3 layer of flight.

v. Installing the flight plan 

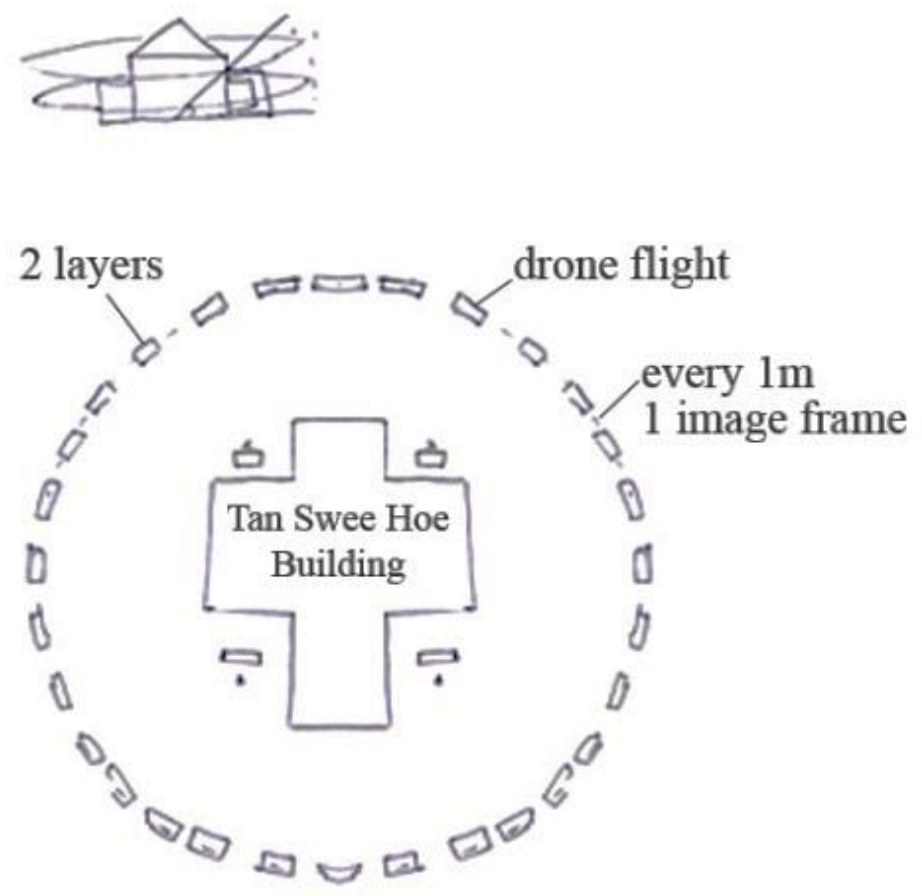

Fig. 3 - Sketch illustrated for flight plan of the drone

The overall ranking of the building will be earned from the assessment of the rating criteria and evaluated using CSP1. Several modes of defect is documented for each side of the building for building structure general, with some of these mechanisms will be observe by the following damage and defect which are most relevant as shown in Fig. 4.

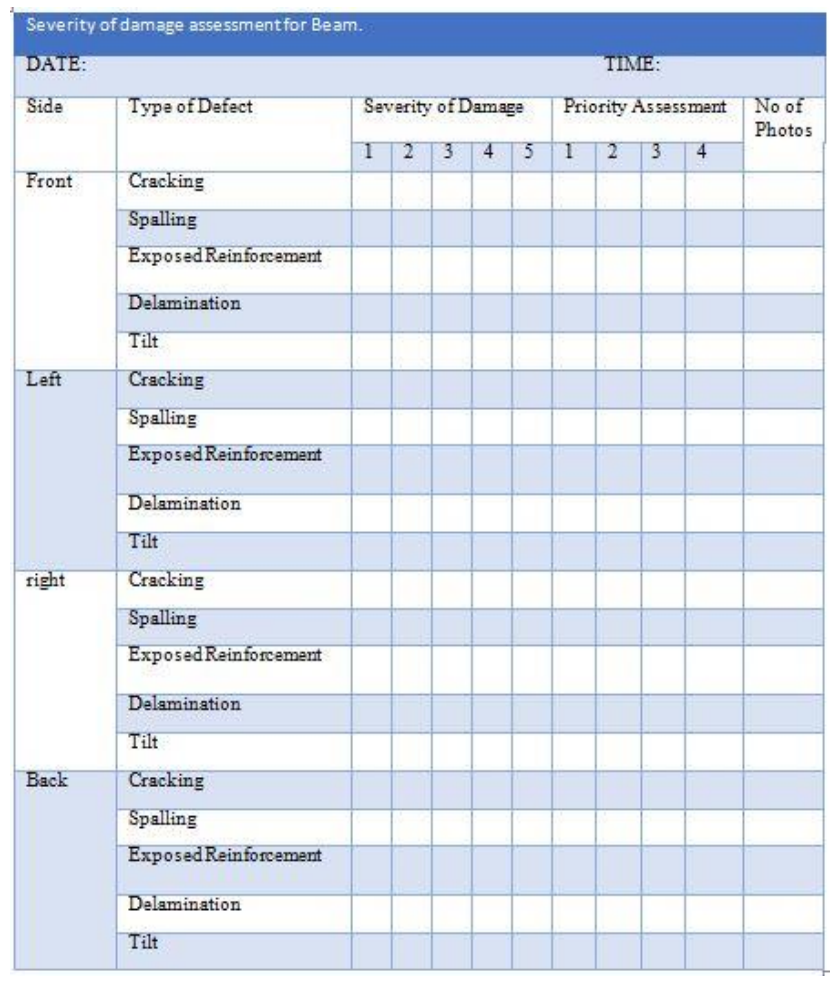

Fig. 4 - Modified assessment form for inspection (PWD, 2002)

From the manual assessment, severity of damage and overall building condition are marked and measured to be analysed at site. Every data is recorded by each individual structural component of the building with severity of damaged ranked on site. Based on the data collected from the $40 \mathrm{~m}$ square Tan Swee Hoe's building, the parameters involved to 
identify the severity of damage and the overall building condition was determined. The condition rating and severity of damage was done by determine the parameters using visual observation from the data collected and analysed. Data from manual building inspection were combined to get the comparison, and can be summarized to conclude the end of results and the effectiveness. The data collected is presented by rank, tables and figures as it is the best ways to summarizing and presenting the result briefly.

\section{Result and Discussion}

The parameters was classified into three distinct categories in order to have better understanding on the overall building condition. First class is 'good' where the building condition are in good quality and does not likely to collapse or can withstand their purpose. The score for good quality falls around 1 to 4 . Otherwise, the second class has been concluded for several reasons which is the building in average condition. The score was range between 5 to 12 . Lastly, the category of dilapidated cases which can be problematic to the building and occupancy of the building.

The severity of damage assessment are divided into two components which are condition assessment and priority assessment. Severity of damage assessment as well as priority assessment were done for Beams, Columns and Walls to detect any type of defect such as Cracking, Spalling, Exposed Reinforcement, Delamination and Tilt for all sides.

From the analysis there are 159 defects for the whole building. From the equation of Condition Survey Protocol (CSP 1), the total value of priority of damage times with severity of damage are 2185 . So when the total value is divided by the total no of defect it is equal to 13.742. Based on the CSP 1, it was categorized that this building is in class dilapidated building. This building requires serious attention to avoid any further damage.

\subsection{Crack Parameters}

An investigation was done at Tan Swee Hoe's building for the inspection of severity of damage ranking parameter which is cracking. This inspection was done by using visualization and categorized the class using form assessment. Fig. 5 shows the severity of damage for cracking which is ranked 2 and priority assessment ranked 1. Both assessment is multiplies giving the value of 2 . The application of matric assessment bringing the severity of damage to category green.

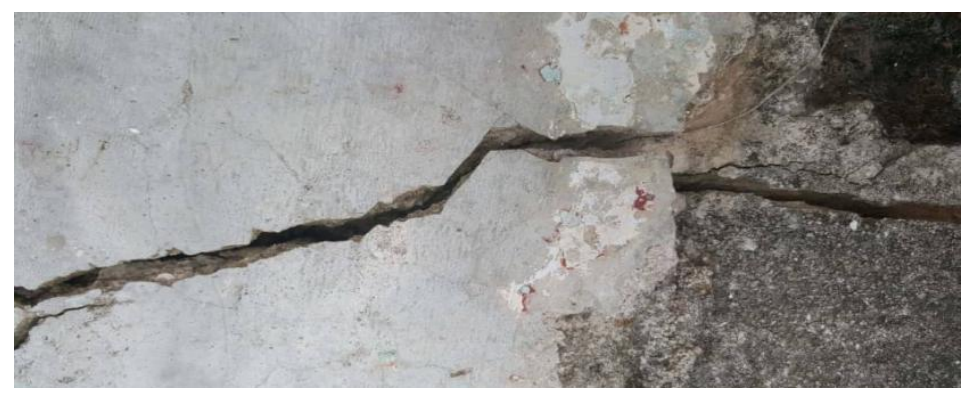

Fig. 5 - Modified assessment form for inspection (PWD, 2002)

As can be seen evaluated from the data, the cracking all around the building are mostly class green which is not require a serious attention. There are also many cracking which categorized class yellow which need a serious attention. There are about $90 \%$ of the cracking class green, $10 \%$ class yellow and no recorded class red cracking for the entire building.

\subsection{Spalling Parameter}

As mentioned in the literature review, several indication related in building defect. One of them is spalling which is investigated from the data collected by using UAV visualization and categorized them into three classes. The most interesting finding was that shown in Fig. 6 whereas the spalling ranked category red which require serious attention. The severity of damage is ranked 5 and priority assessment is 4 . It has the maximum damage available in the matrix assessment system. Fig. 6 shows the picture taken then rendered in Agisoft Photoscan to measure the severity of damage. From average 5 points in red conditions, 25 points in yellow and the rest in green condition. Although majority of spalling is still in good condition which just effect the visual condition of the building. 


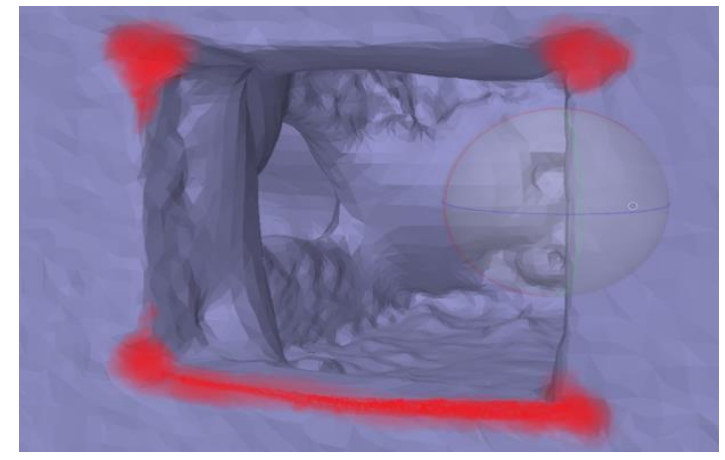

Fig. 6 - Spalling class red located in one of the wall of window frame

\subsection{Exposed Reinforcement Parameter}

Based on the results, overall exposed reinforcement is categorized as class green while few as class red condition. This is mainly occurring at the structural component that carries heavy load such as beam and column. It is tricky to identify the reinforcement due to the material type of reinforcement used for old building plus many of the section areas are covered with mold and vegetation. From the visual inspection, it should be done more carefully by identifying the depth of the exposed reinforcement. Fig. 7 shows the condition of exposed reinforcement on the wall.

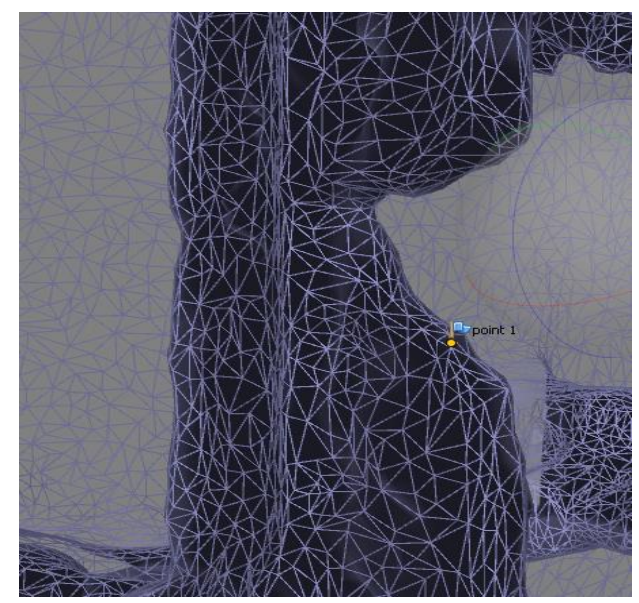

Fig. 7 - Exposed reinforcement from the damage column.

\subsection{Delamination Parameter}

The results show that concrete delaminated is in poor condition. Overall, data indicates that almost every angle of the structure is detected but mainly at class yellow which the area is small but in large volume. As for visual inspection it really turns of the architecture of the building. Delamination cannot be seen from further away as it only can be seen closely. The inspection of delamination part indicate the manual inspection is better and more accurate. The present results as shown in Fig. 8 are in condition monitor, significantly higher than red class.

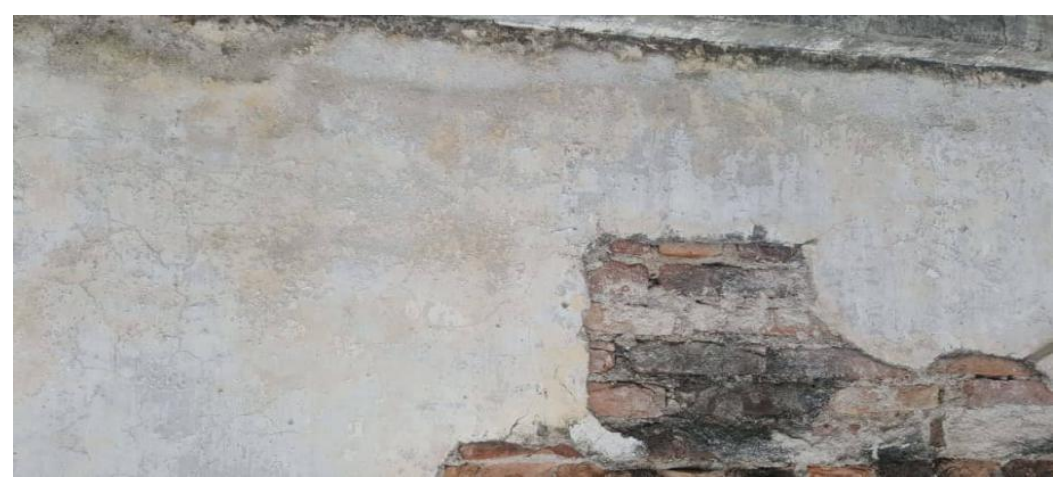

Fig. 8 - Delamination occur after side by side with spalling 


\subsection{Tilt Parameter}

The most difficult finding is to identify if the structure is tilting. Because of the component is mostly dilapidated or spalled half of the structure it is difficult to emerge from the analysis. From the software generated it can be labelled the $\mathrm{y}$ axis and $\mathrm{x}$ axis then the tilt can be seen obviously. From the inspection carried out, only one case which is located at the landmark of the building all the way to column supporting the landmark. It is analyzed as two different condition ranking. The tilt is about less than 10 degree and it has no support to hold the structure. It is evaluated that the severity of damage is rank 5 and priority assessment is 4 which is the maximum the damage can go which is calls red require serious attention. So that, probability for it to collapse are in high level. As we can see in Fig. 9, the degree of tilt can be quite seen with the naked eye.

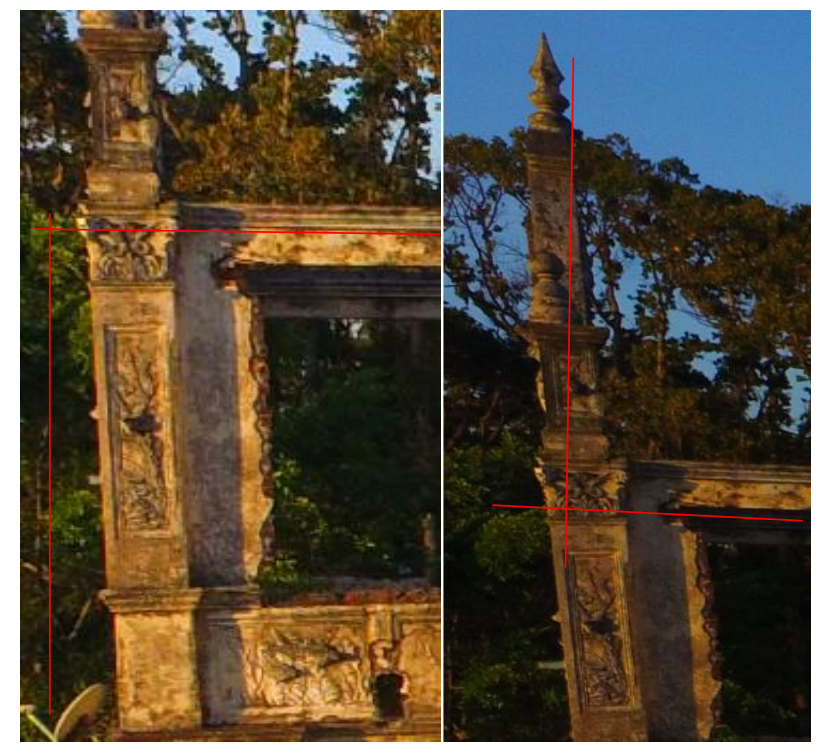

Fig. 9 - Damage of the landmark and column supporting below

\subsection{Measurement}

Regression analysis was used to measure the accuracy of measurement from at site and in the software. Agisoft software rendered the Image and producing a scale of 1:1 to the actual size of the model. From the software the value of a certain point need to be created for the software to compare the value. Fig. 10 we can see the picture of one sample set of data being measured to be compare with in the software. From the comparison it found that the accuracy is up to $10 \mathrm{~mm}$ to the original measurement. For the method of this project the ranking assessment range to 500mm in length and up to $1500 \mathrm{~mm}$ of range data. Fig. 10 below shows the measurement of $350 \mathrm{~mm}$ the length of column while compare to software the measurement is $343 \mathrm{~mm}$. The other comparison is the measurement of window width $2140 \mathrm{~mm}$ on site a $2129.66 \mathrm{~mm}$ in software. To be exact the average accuracy of software is plus minus $10 \mathrm{~mm}$.

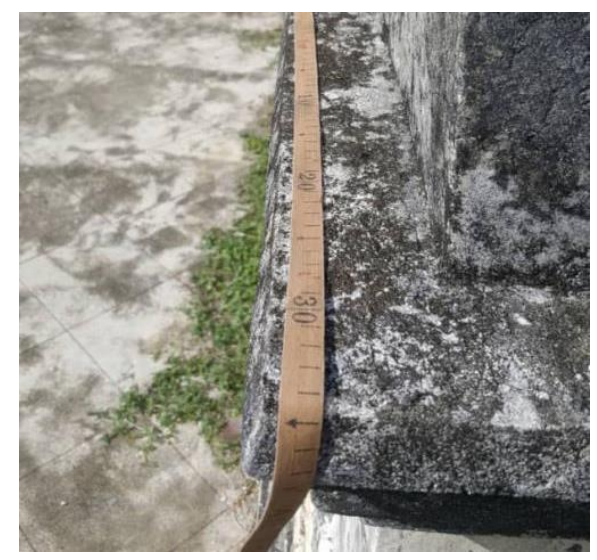

Fig. 10 - Measurement taken at site to be compared with in the software 


\section{Comparison Result}

The comparison from building inspection with UAV assisted and existing manual inspection is in no doubt that it has all the perks individually. As explained by the PWD department the first inspection requires least accurate data but a full covered visual inspection for further work to be adjusted for the building. From table 4.1 we can see the difference between the two methods.

Table 1 - Comparison between manual inspection and UAV assisted inspection

\begin{tabular}{ll}
\hline \multicolumn{1}{c}{ Manual Inspection } & \multicolumn{1}{c}{ Drone assisted Inspection } \\
\hline $\begin{array}{l}\text { Time required on site to personally inspect the defect of } \\
\text { building and severity of damage take up to 1.5-2 hours } \\
\text { depend on the size of building. }\end{array}$ & $\begin{array}{l}\text { Time required to establish ground point and drone to fly } \\
\text { its route takes up to half an hour for the same size } \\
\text { building. }\end{array}$ \\
\hline $\begin{array}{l}\text { Limitation of reach is limited to the tools available } \\
\text { without calling any extra support such as bucket truck. }\end{array}$ & $\begin{array}{l}\text { Drone can reach out to high places with clear image for } \\
\text { inspection. But need to watch out for electrical wiring } \\
\text { and trees. }\end{array}$ \\
$\begin{array}{ll}\text { Also it might be dangerous to reach out for critical place. } \\
\begin{array}{l}\text { The accuracy of manual inspection is very accurate as it } \\
\text { only involve human error and reading measurement error } \\
\text { which only range to 5mm and below. }\end{array}\end{array}$ & $\begin{array}{l}\text { The measurement depends on the many factor include } \\
\text { software generated measurement, data collection, mesh } \\
\text { point and cloud point. It range up to 10mm error. }\end{array}$ \\
\hline $\begin{array}{l}\text { Tools needed for manual inspection is easy to learn and } \\
\text { can be bought any hardware shop. }\end{array}$ & $\begin{array}{l}\text { Require a built in GPS system for location indicator and } \\
\text { require special skill to lean to fly the drone. Also need } \\
\text { the software and drone itself cost quite expensive. }\end{array}$ \\
\hline
\end{tabular}

\section{Conclusion}

The assessment form prepared in this research by referring to PWD guideline and manual, then modified to a closer version to be used in drone inspection. From all parameters measured in the assessment, this research has found that generally Tan Swee Hoe's building were rated in moderate condition. However, the results have also shown that visitors nearby are exposed to quite high risk based on the risk assessment. Since the study was limited by the lack on previous or existing technology regarding to the processed data to be analyzed since it requires a very large computer memory to process such a number of picture. It was not a big issue to not finish this research. Notwithstanding these limitation, the study continued to collect data from site which was evaluated properly. It is because the maximum ability to visually see the picture clearly is when the lighting and the angle of camera pointed is perfect. The perfect time is afternoon since the sunlight is very bright and the weather is not so warm.

\section{Acknowledgement}

Special thanks to the Universiti Tun Hussein Onn Malaysia for making this research possible.

\section{References}

Aadam Mohammed Taha Abdullah. (2019). Historical building inspection based on UAV (Bachelor dissertation, Universiti Tun Hussein Onn Malaysia)

Al-Kaff, A., Moreno, F.M., San José, L.J., García, F., Martín, D., de la Escalera, A., Nieva, A. and Garcéa, J.L.M., 2017, April. Vbii-uav: vision-based infrastructure inspection-uav. In World Conference on Information Systems and Technologies

Banaszek, A., Banaszek, S. and Cellmer, A., (2017). Possibilities of use of uavs for technical inspection of buildings and constructions. In IOP Conference Series: Earth and Environmental Science (Vol. 95, No. 3, p. 032001). IOP Publishing

DJI Phantom. (2015) Phantom Standard. https://www.dji.com/phantom3-stnd

Fisher, M.A., 2015. Integrated sensor suite for indoor building inspection with an unmanned aerial vehicle. Bangor University (United Kingdom) 
Kaamin, M., Idris, N.A., Bukari, S.M., Ali, Z., Samion, N. and Ahmad, M.A., 2017. Visual inspection of historical buildings using micro UAV. In MATEC Web of Conferences (Vol. 103, p. 07003). EDP Sciences

Mader, D., Blaskow, R., Westfeld, P., \& Weller, C. (2016). Potential of Uav-Based Laser Scanner and Multispectral Camera Data in Building Inspection. International Archives of the Photogrammetry, Remote Sensing \& Spatial Information Sciences, 41

Perritt Jr, H. H., \& Sprague, E. O. (2016). Domesticating Drones: The technology, law, and economics of unmanned aircraft. Routledge

Srivastava, A.N., Pasko, D.M., Batla, H., Kantor, I. and Ubhi, G., Verizon Patent and Licensing Inc, (2017). Unmanned aerial vehicle platform. U.S

Tahar, K.N., 2012. Aerial terrain mapping using unmanned aerial vehicle approach. International Archives of the Photogrammetry, Remote Sensing and Spatial Information Sciences, 39, p.B7

Yusof, Hanita, Ibrahim, F.K. and Shahminan, R.N.R.. "Motifs, Placements and Influences of Woodcarving in Muar Traditional Malay Houses.” Advanced Science Letters 23.7 (2017): 6184-6188. Advanced Science Letters. Web 